\title{
Supernova Remnants in Nearby Spiral Galaxies
}

\author{
By KNOX S. LONG
}

Space Telescope Science Institute, 3700 San Martin Drive, Baltimore, MD 21218, USA

Large samples of supernova remnants are needed in order to study the global distribution of supernovae in galaxies, for determining how the environment in which a SN explodes affects the appearance of a SNR, for studying abundances and abundance gradients in galaxies, for estimating SN rates, and in order to determine the energetics of SNRs and their expansion. Here we describe techniques which are currently being used to expand SNR samples in nearby spirals.

\section{Introduction}

The Cygnus Loop is thought to be about 18,000 years old (Ku et al. 1984). Assuming a SN rate of 5 per century (van den Bergh \& Tammann 1991), there should be about $900 \mathrm{SNR}$ in the galaxy younger than the Cygnus Loop. However, Green's revised catalog of Galactic SNRs contains $182 \mathrm{SNRs}$, some of which are clearly more evolved than the Cygnus Loop (Green, these proceedings). As a result, it is clear that the Galactic sample is very incomplete. In the Galaxy, nearly all SNRs have been first recognized as SNRs from radio observations. Since SNRs are found primarily in the Galactic plane and since $\mathrm{X}$-rays and optical light are strongly absorbed by material in the Galactic plane, they are hard to detect in these wavelength bands. In fact, only about 40 Galactic SNRs have been detected at optical wavelengths and only about 50 have been detected at X-ray wavelengths. (However, see Aschenbach [these proceedings] who suggests there may be a population of X-ray bright, radio faint SNRs hidden in the ROSAT survey data.)

Various attempts (e.g. Li et al. [1991]; Green [these proceedings]) have been made to use the Galactic sample of SNRs to determine the radial distribution of SNRs in the Galaxy, the association of SNRs with spiral arms, the progenitor populations of Galactic SNRs, and the SN rate. However, if large samples of extragalactic samples can be assembled, they are likely to be far better samples for answering these kinds of questions (in other galaxies) - because the distances to all the SNRs in a sample will be identical, and because extinction and confusion are less severe in face-on spirals. Extinction along the line of sight to a SNR affects not only basic detectability, but also appearance, which is very important if one hopes to classify the SNRs in a sample. It is also more straightforward to estimate total fluxes from extragalactic SNRs since they have small angular sizes, although this comes at a price, since small size means accurate diameters are difficult to obtain.

The first successful attempt to identify a significant number of SNRs -12 - in a nearby galaxy - the Large Magellanic Cloud - was made by Mathewson \& Clarke (1973). For this survey, they defined a SNR as an emission nebulae which had a [S II] $\lambda \lambda 6717,6731: \mathrm{H} \alpha$ ratio large compared to that observed in $\mathrm{H}$ II regions and which was associated with a nonthermal radio source.

In the late 1970's and early 1980's, a number of observers, notably D'Odorico et al. (1980), Blair, Kirshner, \& Chevalier(1981), Dopita et al. (1984) and Blair \& Kirshner (1985), carried out optical interference surveys of nearby galaxies, identifying of order 40 SNRs in Local Group spirals. Radio surveys were also attempted by Dickel \& D'Odorico 
(1984), Goss et al. (1980), Reynolds \& Fix (1987), and others, but only a small number $(\sim 20)$ of SNRs were detected and the ones which were detected were some of the same SNRs previously identified as optical SNRs.

In the same time period, the Einstein Observatory was used to make the first imaging X-ray surveys of the Magellanic Clouds and nearby spirals. Long et al. (1981) identified 25 SNRs in the LMC, including 4 new SNRs with Balmer-dominated optical spectra (Tuohy et al. 1982). Inoue, Koyama \& Tanaka (1983) identified 6 SNRs in the SMC, including 1E0102-72, a new oxygen-dominated SNR (Dopita, Tuohy \& Mathewson 1981). These surveys were important because they constituted the largest "homogeneous" X-ray samples of SNRs. However, no new SNRs were identified in Local Group spirals, and only a few were detected.

With the advent of new detector technologies - CCDs with linear response functions and high quantum efficiencies, radio telescopes on which multiple frequencies can be observed with the same angular resolution, and X-ray telescopes with higher sensitivityit has become possible to carry out observations which greatly increase the numbers of SNRs that are likely to be detected. I would like to use the remainder of this review to describe some of these observations.

\section{Optical Searches for SNRs}

Optically, most SNRs are extended emission line objects. Because the cooling time behind the shock is short compared to age of the SNR, the spectra which are observed show strong lines from a wide range of ionization states. This distinguishes SNRs from $\mathrm{H}$ II regions, because in $\mathrm{H}$ II regions, photoionization maintains the bulk of the plasma in well-defined ionization states. The most common diagnostic is the [S II] $\lambda \lambda 6717,6731$ : $\mathrm{H} \alpha$ ratio which is typically of order $0.6-1.2$ in SNR shocks and of order 0.1 in bright $\mathrm{H}$ II regions. (In H II regions, S is mostly double ionized and therefore [S II] $\lambda \lambda 6717,6731$ is weak.)

The original optical searches for SNRs were made using interference filters with photographic plates as the recording material. Recently, we (W.P Blair [JHU], R. P Kirshner [Harvard College], P.F. Winkler [Middlebury College] and I ) have carried out new interference filter surveys using CCDs cameras at Kitt Peak and CTIO and on the $2.5 \mathrm{~m}$ at Las Campanas. All of these surveys consist of obtaining images in [S II], $\mathrm{H} \alpha$, and a nearby continuum band. The analysis of the first of these surveys, which covered the inner 15' of M33, revealed 30 new SNRs (where previously there had been 10) (Long et al. 1990; Smith et al. 1993). In addition to acquiring data which covers a much larger portion of M33, we have now surveyed a number of other galaxies including M83, NGC 247, NGC 300, NGC 6946 and NGC 7793. Our analyses of these data are still underway, but it is quite clear that 10-45 SNRs are identifiable in each galaxy, including the most distant NGC 6946. The results on NGC 300 are illustrative (Long \& Blair 1994):

NGC 300 is a nearby Sculptor group spiral; most recent distance estimates lie between 1.5 and $2 \mathrm{Mpc}$. Following Deharving et al. (1988), we will assume $1.53 \mathrm{Mpc}$, which means it is about twice as far as M33. Because of its proximity and its low inclination angle $\left(42.3^{\circ}\right)$, it is an excellent galaxy for SNR searches, especially since it lies at high galactic latitude which means Galactic absorption is small. Dopita, D'Odorico \& Benvenuti (1980) carried out the only previous search for SNRs in NGC 300; they used H $\alpha$ and [S II] to identify 7 candidates. Two of these objects - DDB2 and DDB5 - have been confirmed as SNRs based on optical spectroscopy.

We observed 9 fields in NGC 300, each 5.3' on a side with $\mathrm{H} \alpha$, [S II], and $6100 \AA$ continuum filters using the 2.5 meter at Las Campanas. The dividing line between a SNR 


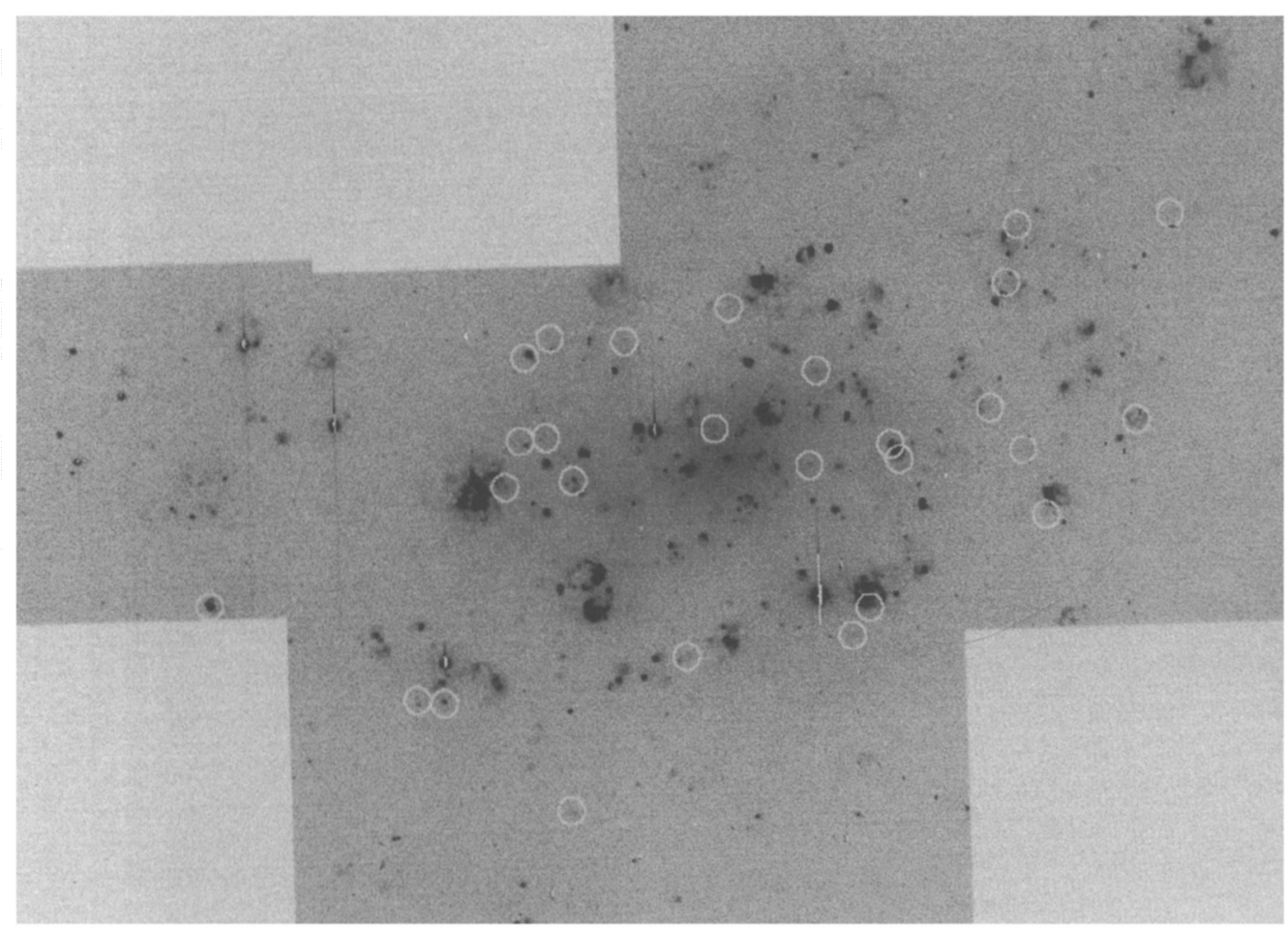

FIgURE 1. A mosaic of the $\mathrm{H} \alpha$ images of NGC 300 with SNRs identified.

and an $\mathrm{H}$ II region has usually been taken to be a [S II] : $\mathrm{H} \alpha$ ratio of 0.4 (D'Odorico 1978). Therefore, these data were searched for nebulae with [S II] : $\mathrm{H} \alpha$ ratios exceeding this value with little or no continuum contamination. A total of 27 potential SNRs were identified in this way.

The distribution of the SNRs is shown on the mosaic of the $\mathrm{H} \alpha$ images in Fig. 1. The faintest objects which were identified have $\mathrm{H} \alpha$ surface brightnesses of about $7 \times$ $10^{-16} \mathrm{ergs} \mathrm{cm}^{-2} \mathrm{~s}^{-1} \operatorname{arcsec}^{-2}$. Five of the 7 objects identified by Dopita, D'Odorico, \& Benvenuti as SNRs were within our survey region. Only two of these - DDB2 and DDB5 - were identified as SNR candidates in our survey; the other three - DDB1, DDB4, and DDB7 - are present in our images but are dominated by continuum emission. Dopita, D'Odorico, \& Benvenuti did not have continuum images, and therefore it is not surprising that some of the objects in their list are not SNRs. DDB2 and DDB5 have surface brightnesses 30 times that of the faintest objects in our survey.

In order to confirm that the nebulae which we had identified were SNRs, we subsequently obtained spectra of about $2 / 3$ of our sample. All of the candidates we observed did indeed have $[\mathrm{S} \mathrm{II}]: \mathrm{H} \alpha$ line ratios greater than 0.4 , and the higher surface brightness candidates generally showed emission from [O I] $\lambda \lambda 6300,6363$ (which is exceedingly faint in $\mathrm{H}$ II regions). Since our confirmation rate was in fact $100 \%$, we are confident that all 27 of the nebulae satisfy the conventional optical definition for a shock heated nebula.

Inspection of Fig. 1 shows that a significant fraction of the SNRs in NGC 300 are associated with the spiral arms of the galaxy. The surface density of SNRs (in our sample) decreases from about $1 \mathrm{kpc}^{-2}$ at a galactocentric radius of $1 \mathrm{kpc}$ to about 0.1 $\mathrm{kpc}^{-1}$ at $4 \mathrm{kpc}$. Except for an overall scale factor, the variation of the surface density resembles that of the $H$ II regions in the catalog compiled by Deharving et al. (1988). 
There are $22 \mathrm{SNRs}$ in our sample with a diameter of $50 \mathrm{pc}$ or less. The $\mathrm{N}(<\mathrm{D})$ vs. D relationship has a maximum likelihood power law slope of $2.9 \pm 0.8$ which is close to the value of 2.5 expected from a Sedov expansion law. If we assume our sample is complete to $50 \mathrm{pc}$ and that the ratio of the explosion energy to the ISM density $\mathrm{E} / \mathrm{n}$ is $10^{51} \mathrm{ergs} \mathrm{cm}^{3}$, then SNe occur in NGC 300 once every 2300 years. This implies a SN rate of $0.3 \mathrm{SNe}(100 \mathrm{yr})^{-1}\left(10^{10} \mathrm{~L}_{\odot}\right)^{-1}$, a factor of 3 lower than derived by Long et al. (1990) for M33.

\section{Radio Searches for SNRs}

Radio searches for SNRs have been based upon the same criteria as have searches for Galactic SNRs, where the observational definition for a SNR is an extended, typically limb-brightened source with a non-thermal radio spectrum. As at optical wavelengths, $\mathrm{H}$ II regions constitute the principle source of confusion (especially for pulsar-dominated SNRs which have flatter spectra). Because the apparent sizes of SNRs are small (5-10" in M33 for example), early surveys were hampered by the fact that beam sizes differed at different wavelengths. Scaled array observations (or more correctly, observations from which multi-frequency maps with the same angular resolution can be constructed) are required in order to obtain accurate spectral indices.

The power of this technique has been best demonstrated by Duric et al. (1993) who studied M33. Duric et al. used the VLA and Westerbork to obtain continuum images of M33 at 6 and $20 \mathrm{~cm}$ with a resolution of about 7". The rms noise ratio of the images was about $50 \mu \mathrm{Jy}$ at both frequencies, roughly 1000 times smaller than the expected flux from a SNR like Cas A at the distance of M33. Duric et al. concentrated on 53 optically-identified SNRs in M33, which they took from the survey carried out by Long et al. (1990), supplemented by 10 optically-confirmed SNRs outside the region studied by Long et al. Using a method which involved searching for excess emission at $20 \mathrm{~cm}$ (over that observed at $6 \mathrm{~cm}$ ), Duric et al. detected nonthermal emission from 26 of the 53 optically-identified SNRs. However, the brightest of these SNRs, DDB9, is considerably fainter $\left(\mathrm{S}_{\nu}(30 \mathrm{~cm})=5.1 \mathrm{mJy}\right)$ than Cas A would be at the distance of M33 $(45 \mathrm{mJy})$. In fact, there are approximately 20 Galactic SNRs which are more luminous than the brightest SNR in M33.

S. Gordon and his collaborators (these proceedings) are continuing to analyze the new radio data on M33 and are comparing it to an expanded sample of 72 optically-identified SNRs in M33. There appear to be approximately 110 nonthermal sources in the field. Thirty-eight of these are spatially coincident with optically-identified SNRs. About 30 of the nonthermal sources in the field are likely to be background galaxies at the flux limit of the radio survey. Therefore, it is possible that there are as many as 80 radio-detected SNRs in the survey.

One of the nonthermal sources in M33 is coincident with the giant $\mathrm{H}$ II region NGC 592. Gordon et al. (1993) used the position of the nonthermal source to identify a portion of the nebula with a high [S II]:H $\alpha$ ratio and subsequently obtained optical spectra which confirm it is a SNR. The new source is also coincident with a soft Einstein (and ROSAT) $\mathrm{X}$-ray source.

\section{X-ray Searches for SNRs}

X-ray surveys of SNRs in external galaxies are important because most of the energy in shock-dominated SNRs is contained in the hot thermal plasma which produces X-ray emission. SNRs are the only extended sources in galaxies with soft X-ray spectra and 


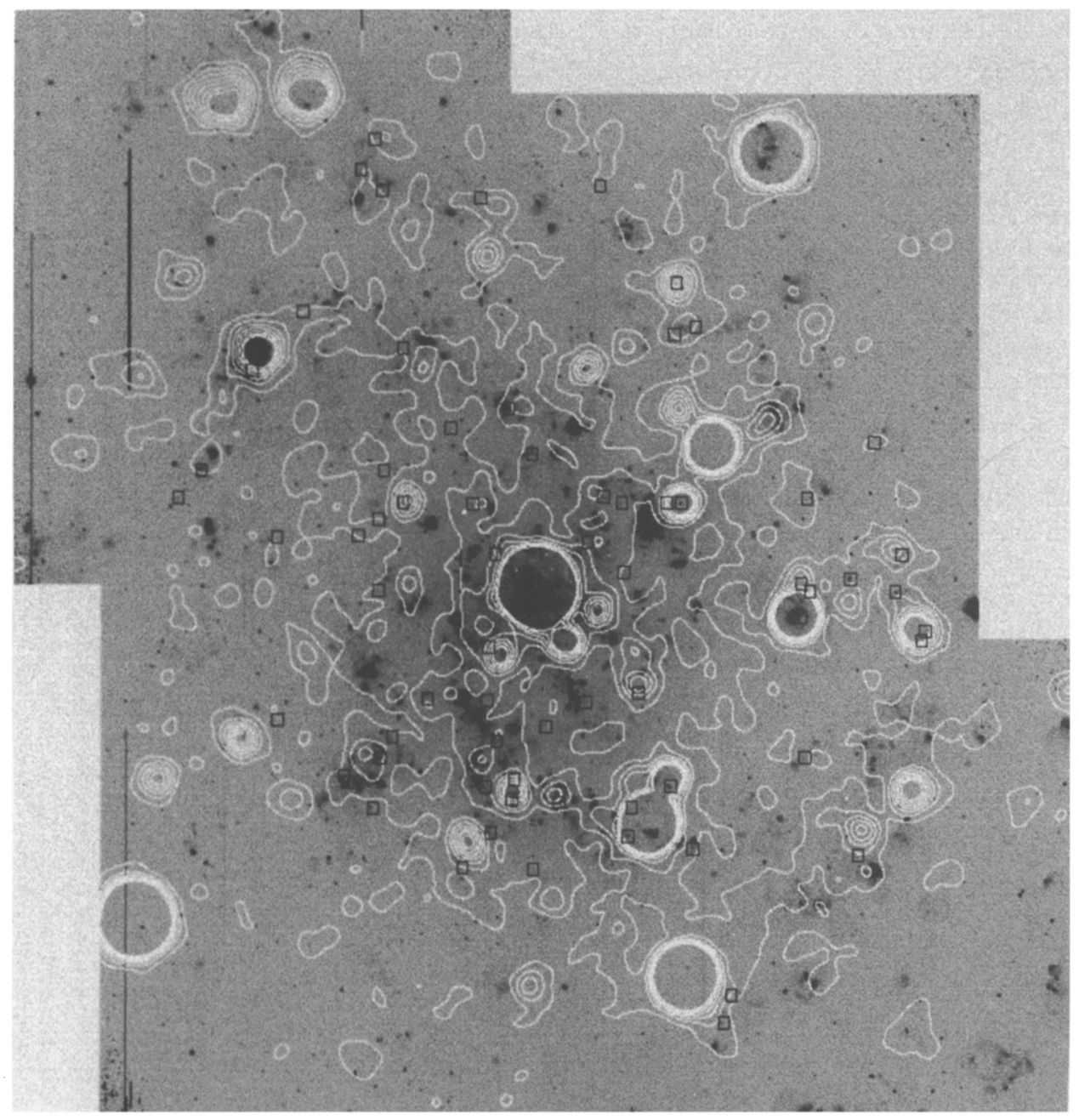

Figure 2. An X-ray contour map of M33 superposed on an $\mathrm{H} \alpha$ mosaic. The positions of our current working list of 72 optically-identified SNRs are also indicated.

luminosities in excess of $10^{34} \mathrm{ergs} \mathrm{s}^{-1}$. An advantage of X-ray searches over searches at other wavelengths is that $\mathrm{H}$ II regions do not compromise one's ability to identify an object as a SNR. In principle, therefore, X-ray observations provide a powerful way to search for SNRs in nearby galaxies.

The launch of the first imaging X-ray telescope, Einstein made it possible to carry out searches for SNRs in nearby galaxies. As noted above, the searches succeeded but primarily in the Magellanic Clouds. ROSAT, with a larger effective area, lower background rate, and improved imaging performance, is now enabling searches with about an order of magnitude more sensitivity than Einstein. Even so, SNR searches are likely to remain difficult. Except in the Magellanic Clouds, it will be extremely difficult to detect SNRs with the HRI, and therefore it will be hard to determine whether a candidate source is extended. As a result, most SNR searches will rely on a more problematic attempt to identify SNRs as soft X-ray sources. This introduces confusion since some binary X-ray sources have soft spectra. Therefore, firm identification of a source as a SNR will require confirmation using optical or radio tests. 
As an example of what is likely to be accomplished, however, let us consider M33. Because of its proximity (840 kpc; Freedman, Wilson \& Madore 1991), relatively low inclination angle $\left(57^{\circ}\right.$; Searle 1971), and well-defined open spiral arms, M33 is an ideal galaxy for studying the X-ray properties of spiral galaxies, including their SNR populations. As a result it was observed with the IPC and HRI on Einstein (Long et al. 1981; Markert et al. 1983; Trinchieri, Fabbiano \& Peres 1988). These studies revealed a dominant nuclear source, the brightest in the Local Group $\left(L_{x} \sim 10^{39}\right.$ ergs s$\left.^{-1}\right)$ and 14 other sources $\left(\mathrm{L}_{\mathrm{x}} \geq 10^{37} \mathrm{ergs} \mathrm{s}^{-1}\right)$ thought to be associated with M33. Of these sources, 3 are positionally coincident with known optical SNRs in M33.

$\mathrm{X}$-ray contours from a $50 \mathrm{ksec}$ exposure with the PSPC on ROSAT are shown in Fig. 2 superposed on an $\mathrm{H} \alpha$ mosaic obtained as part of the expanded optical search for SNRs in M33. There are about 31 individual sources in the image within 15' of the nucleus of M33. All of the bright sources which had been detected with Einstein are visible. Many of the sources are clearly associated with H II regions. Diffuse emission (perhaps simply a superposition of low luminosity sources) can be traced along the northern and southern spiral arms. Also shown on the Figure are the positions of our current working sample of optically-identified SNRs in M33.

In an attempt to determine how many of these sources are SNRs we have examined the positional coincidences between X-ray sources and optically-identified SNRs. Ten of the 31 X-ray sources lie within 30" of known SNRs in M33. If one assumes that both source populations are uniformly distributed over M33, one would expect to find only two chance coincidences, which suggests either that there are a number of SNRs in the $\mathrm{X}$-ray sample or alternatively that both SNRs and X-ray sources have similar parent populations.

ROSAT has a relatively limited spectral range - 0.1-2.4 keV. M33 sources are also affected by galactic absorption of about $6 \times 10^{20} \mathrm{~cm}^{-2}$, which strongly absorbs X-rays below $0.6 \mathrm{keV}$. Nevertheless, in an attempt to separate the sources according to their $\mathrm{X}$-ray spectra, we have constructed hardness ratios, defined as $(\mathrm{H}-\mathrm{S}) /(\mathrm{H}+\mathrm{S})$ where $\mathrm{H}$ is the count rate above $1 \mathrm{keV}$ and $\mathrm{S}$ is the count rate below $1 \mathrm{keV}$, for all the point sources. A histogram of the hardness ratios we obtained is shown in Fig. 3. Eight of the eighteen sources with hardness ratios less than 0.0 lie within 30 " of a SNR. This suggests that we have in fact detected X-ray emission from at least 8 SNRs, that it may be possible to identify candidate SNRs on the basis of their X-ray spectra, and (more speculatively) that a substantial fraction of the other soft sources are SNRs. Obviously, detailed spectroscopic studies are required to verify these suggestions.

\section{Conclusion}

It is now possible to assemble relatively homogeneous large samples of SNRs for galaxies as distant as $10 \mathrm{Mpc}$. In galaxies, such as M31 and M33, the numbers of SNRs which can be detected (at optical wavelengths) will be comparable to the numbers of SNRs known in the Galaxy. Although the selection effects are clearly not yet fully understood, these samples should allow us to understand a great deal more about the distribution of SNRs and hence $\mathrm{SNe}$ in galaxies than is possible with the current Galactic sample.

\section{Acknowledgments}

Very few of the programs I have described here would have been possible without the diligence of my collaborators - W. P. Blair, N. Duric, S. Gordon, R. P. Kirshner, R. 


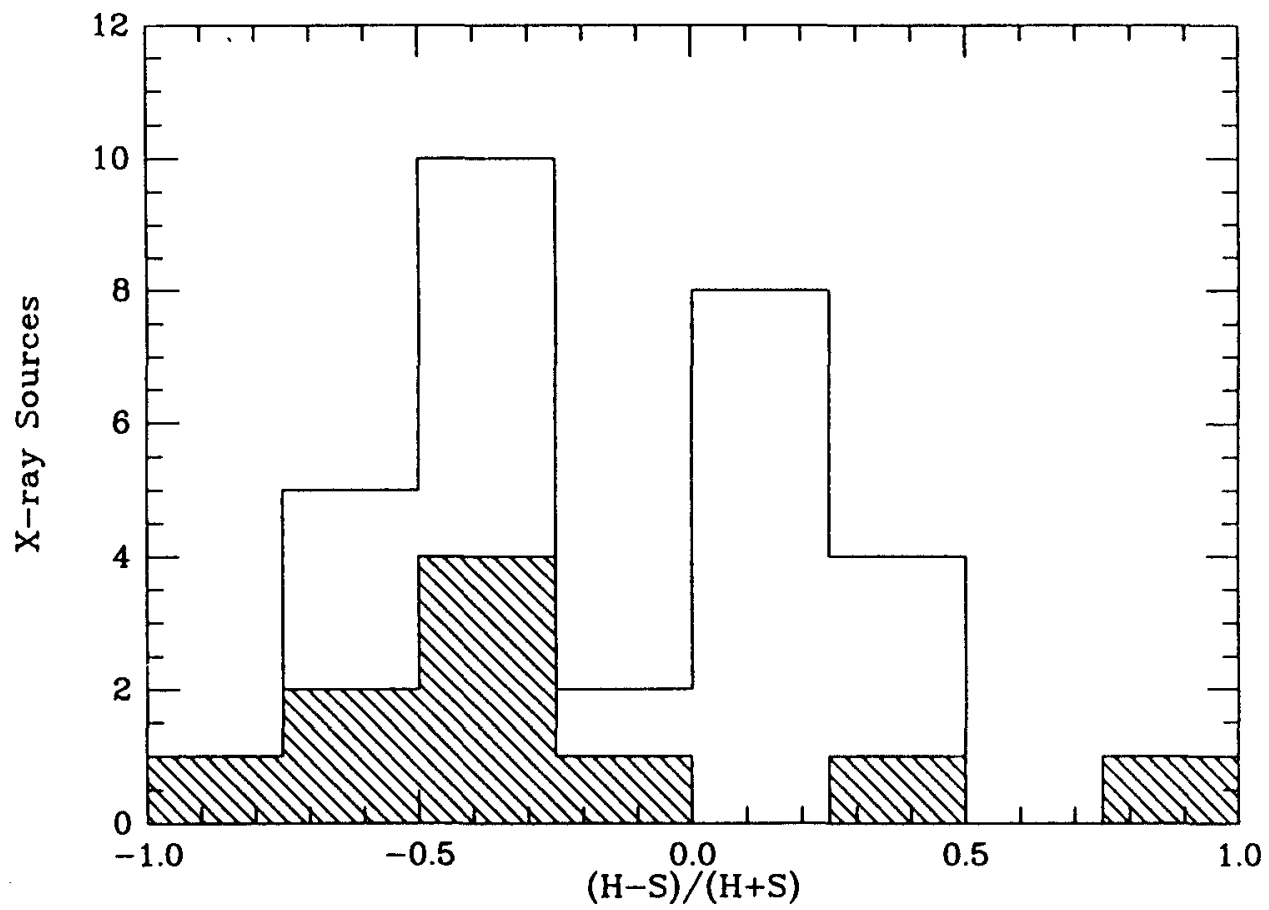

FIGURE 3. Hardness ratios of the 31 point sources within 15' of the nucleus of M33 as observed with the ROSAT. X-ray sources which are positionally coincident with optically-identified SNRs are shaded.

C. Smith, and P. F. Winkler. This work has been supported in part by NASA grant NAG5-1539.

\section{REFERENCES}

Blair, W. P., \& Kirshner, R. P. 1985, ApJ, 289, 582

Blair, W. P., Kirshner, R. P., \& Chevalier, R. A. 1981, ApJ, 247, 879

Deharving, L., Caplan, J., Leqeaux, J., Azzopardi, M., Bresacher, J, Terengi, M., \& Westerlund B. $1988, A \& A S, 73,407$

Dickel, J. R., and D'Odorico, S. 1984, MNRAS, 206, 351

D'Odorico, S. 1978, Mem. Soc. Astr. Italiana, 49, 563

Dopita, M. A., Binnette, L., D'Odorico, S., and Benvenuti, P. 1984, ApJ, 276, 653

Dopita, M. A., D'Odorico, S., \& Benvenuti, P. 1980, ApJ, 236, 628

Dopita, M. A., Tuohy, I. R., \& Mathewson, D. S., ApJ, 248, L105

Duric, N., Viallefond, F., Goss, W. M., and van der Hulst, J. M. 1993, A\&AS, 99, 217

Freedman, W. L., Wilson, C. D., \& Madore, B. F. 1991, ApJ, 372, 455

Gordon, S. M., Kirshner, R. P., Duric, N., \& Long, K. S. 1993, ApJ, 418, 743

Goss, W. M., Ekers, R. D., Danziger, I. J., and Israel, F. P. 1980, MNRAS, 193, 901

Inoue, H., Koyama, K., \& Tanaka, Y. 1983, in "IAU Symposium No. 101 Supernova Remnants and Their X-ray Emission", ed. by J. Danziger \& P. Gorenstein (Dordrecht: Reidel Publ. Co.), pp. 535

Ku, W. H-M, Kahn, S. M., Pisarski, R., \& Long, K. S. 1984, ApJ, 278, 615 
Li, Z., Wheeler, J. C., Bash, F. N., Jefferys, W. H. 1991, ApJ, 378, 93

Long, K. S., \& Blair, W. P. 1994, in preparation.

Long, K. S., Blair, W. P., Kirshner, R. P., \& Winkler, ApJS, 72, 61

Long, K. S., D'Odorico, S., Charles, P. A., \& Dopita, M. A. 1981, ApJ, 246, L61

Long, K. S., Helfand, D. J, \& Grabelsky, D. A., ApJ, 248, 925

Markert, T. H., \& Rallis, A. D. 1983, ApJ, 275, 571

Mathewson, D. S., \& Clarke, J. N. 1973, ApJ, 180, 725

Reynolds, S. P. \& Fix, J. D. 1987, ApJ, 322, 671

Searle, L. 1971, ApJ, 168, 327

Smith, R. C., Kirshner, R. P., Blair, W. P., Long, K. S., \& Winkler, P. F. 1993, ApJ, 407, 564

Trinchieri, G., Fabbiano, G., \& Peres, G. 1988, ApJ, 325, 531

Tuohy, I. R., Dopita, M. A., Mathewson, D. S., Long, K. S., \& Helfand, D. J. 1982, ApJ, 261, 485

van den Bergh, S., \& Tammann, G. A. 1991, ARAA, 29, 363 